\title{
Analytical calculation of the longitudinal space charge and resistive wall impedances in a smooth cylindrical pipe
}

\author{
Ahmed M. Al-khateeb* \\ Department of Physics, Faculty of Science, Yarmouk University, Irbid, Jordan \\ Oliver Boine-Frankenheim and Ingo Hofmann \\ Hochstromstrahlphysik, Geselschaft für Schwerionenforschung m.b.H. (GSI) Darmstadt, Planckstrasse 1, D-64291 Darmstadt, Germany \\ Giovanni Rumolo \\ Accelerator Physics Group, SL Division, CERN, CH-1211 Geneva 23, Switzerland \\ (Received 22 September 2000; published 25 January 2001)
}

\begin{abstract}
The longitudinal space charge and resistive wall impedances have been investigated in a smooth cylindrical beam pipe. At any point from the beam axis, we obtained an expression for the total impedance, which at the beam surface $r=a$ for infinite pipe wall conductivity gives the expression for the total impedance that was derived by Zotter and Kheifets in studying the impedance of uniform beams in concentric cylindrical wall chambers, when a single cylindrical chamber is considered [B. W. Zotter and S. A. Kheifets, Impedances and Wakes in High-Energy Particle Accelerators (World Scientific, Singapore, 1998), Chap. 6]. A fitting formula for the space-charge impedance at the beam surface $(r=a)$, which is valid for arbitrary wavelengths, is given. Rather than calculating the impedance with the field on the axis [Joseph J. Bisognano, Fifth European Particle Accelerator Conference (EPAC96), edited by S. Myers, A. Pacheco, R. Pascual, Ch. Petit-Jean-Genaz, and J. Poole (Institute of Physics, Bristol, 1996), Vol. 1, p. 328], the fitting formula is obtained by averaging over the transverse beam distribution. We also give another approach for the calculation of the resistive wall impedance using the flux of the Poynting vector at the pipe wall and then compare it with the expression obtained from the volume integral over the beam distribution.
\end{abstract}

DOI: 10.1103/PhysRevE.63.026503

PACS number(s): 29.27.Bd, 29.20.-c, 47.27.Vf, 85.30.Fg

\section{INTRODUCTION}

The term impedance was first used by Heaviside in the 19 th century to describe the complex ratio of voltage to current $V / I$ in ac circuits consisting of resistors, inductors, and capacitors, see Ref. [1]. In the 1930s, Schelkunoff recognized that the impedance concept could be extended to electromagnetic fields in a systematic way and noted that impedance should be regarded as characteristic of the type of field as well as of the medium [1]. The concept of impedance forms an important link between field theory and circuit theory $[2,3]$.

Wave impedances defined by the ratio of the transverse electric and magnetic fields $Z_{w}=E_{t} / H_{t}$ are characteristics of the particular type of waves. Transverse electromagnetic waves (TEM), transverse magnetic waves (TM), and transverse electric waves (TE) each have different wave impedances $\left(Z_{\mathrm{TEM}}, Z_{\mathrm{TM}}, Z_{\mathrm{TE}}\right)$, which may depend on the type of line or guide, the material, and the operating frequency. The intrinsic impedance of a particular medium $\eta=\sqrt{\mu / \epsilon}$ is dependent only on the material parameters of the medium, but is equal to the wave impedance for plane waves [4].

The ratio of voltage to current for traveling waves is known as the characteristic impedance $Z_{c}=V / I$. Since voltage and current are uniquely defined for TEM waves, the

\footnotetext{
*Author to whom correspondence should be addressed. Email address: helga@yu.edu.jo
}

characteristic impedance of a TEM wave is unique. The TE and TM waves, however, do not have a uniquely defined voltage and current, so the characteristic impedance for such waves may be defined in various ways. At microwave frequencies the measurement of voltage or current is difficult (or impossible), unless a clearly defined terminal pair is available. Such a terminal pair may be present in the case of TEM-type lines (such as coaxial cable, microstrip, or stripline), but does not exist for non-TEM lines (such as rectangular, circular, or surface waveguides) [4].

The concept of coupling impedance in accelerators, which is equivalent to the characteristic impedance $Z_{c}$, was first introduced for the studies of instabilities in the ISR at CERN $[5,6]$. In the design of accelerators it is desired to reduce the coupling impedance of the beam to its environment in order to prevent beam instabilities. Concerning the longitudinal dynamics of charged particle beams there are two important physical quantities, the longitudinal space charge, and the resistive wall impedances [6-14]. A coasting beam of charged particles excites electromagnetic fields in its environment. Depending on the coupling of the beam to its environment at a particular frequency, periodic excitations occur. These excitations perturb the beam dynamics and lead to beam instabilities [13-22].

The coupling impedance of straight, uniform beams in a concentric, cylindrical vacuum chamber, whose walls consist of many layers of different materials was treated by Zotter and Kheifets [7,8]. We find by Zotter and Kheifets an expression for the total impedance at the beam surface $r=a$, which does not give the impedance at any point $r$ from the 
beam axis. Kurennoy and Wang reviewed the definition of the longitudinal space-charge impedance and the corresponding geometry factors for smooth chambers of perfectly conducting walls in the long-wavelength approximation $[10,13]$. Wang et al. determined experimentally the geometry factor for longitudinal perturbations in a space-charge dominated beam, and found that the geometry factor obeys the relation $g=2 \ln (b / a)$, where $a$ and $b$ are the beam and pipe radii, respectively [17].

Bisognano has recently investigated solitary waves in nonrelativistic particle beams [23]. A fitting formula for the ratio of the Fourier-transformed potential and density was proposed, which is exactly the expression for the geometry factor of the longitudinal coupling impedance of a pipe of infinite wall conductivity. The factor

$$
\frac{1+2 \log (b / a)}{1+\left(k^{2} a^{2} / 4\right)[1+2 \log (b / a)]}
$$

in Bisognano's fitting formula, that was used by Rumolo et al. to fit the space-charge impedance for all wavelengths [22], needs some modifications when calculating the impedance by averaging over the transverse beam distribution rather than using the field on the axis, and when accounting for relativistic effects.

In this paper we present the calculation of both the space charge and the resistive wall impedances for all wavelengths and give expressions for the corresponding generalized and approximate geometry factors. For nonrelativistic particle beams with a finite size, these physical quantities are of importance for the longitudinal beam dynamics and for the longitudinal beam instability analysis.

The paper is organized as follows: In Sec. II we present the derivation of the electromagnetic fields in a beam pipe of both infinite and finite wall conductivities. In Sec. III we calculate the space-charge impedance for a pipe of infinite wall conductivity and give a fitting formula with some values for the fitting parameters of the corresponding generalized geometry factor. In Sec. IV we calculate, in a consistent way, the space-charge and resistive wall impedances for a pipe of finite wall surface impedance, and then confirm our expression for the resistive wall impedance by recalculating it from the flux of the Poynting vector over a closed surface surrounding the beam. In Sec. V, we present our numerical analysis, and finally in Sec. VI our conclusions.

\section{ELECTROMAGNETIC FIELDS IN A CYLINDRICAL PIPE}

Upon using Faradays and Amperes laws, the wave equations satisfied by the magnetic $\vec{B}$ and electric $\vec{E}$ fields are

$$
\begin{gathered}
\nabla^{2} \vec{B}-\frac{1}{c^{2}} \frac{\partial^{2} \vec{B}}{\partial t^{2}}=-\mu_{0} \vec{\nabla} \times \vec{j}, \\
\nabla^{2} \vec{E}-\frac{1}{c^{2}} \frac{\partial^{2} \vec{E}}{\partial t^{2}}=\mu_{0} \frac{\partial \vec{J}}{\partial t}+\frac{\vec{\nabla} \rho_{c}}{\epsilon_{0}},
\end{gathered}
$$

where $\rho_{c}$ and $\vec{j}$ are the charge and the current densities, respectively, which obey the following continuity equation:

$$
\frac{\partial \rho_{c}}{\partial t}+\vec{\nabla} \cdot \vec{j}=0
$$

Finite and infinite pipe wall conductivities will be taken into account as imposed boundary conditions on the electromagnetic fields excited by the beam. Assuming that the beam is moving in a cylindrical pipe of radius $b$ with a constant longitudinal velocity $\vec{v}=\beta c \hat{z}$ along the $z$ axis, we represent its charge and current densities by the following relations:

$$
\begin{gathered}
\rho_{c}(\vec{r}, t)=\rho_{c}(r) \delta(z-v t), \\
\vec{j}(\vec{r}, t)=\rho_{c}(\vec{r}, t) \vec{v}=\rho_{c}(r) \beta c \delta(z-v t) \vec{z},
\end{gathered}
$$

where $\rho_{c}(r)$ is an axially symmetric transverse beam distribution. The total charge $Q$ associated with the charge distribution $\rho_{c}(\vec{r}, t)$ in Eq. (4) is

$$
Q=2 \pi \int_{0}^{a} \rho_{c}(r) r d r .
$$

Upon Fourier transformation of Eq. (1) and Eq. (2) in time, we get

$$
\begin{gathered}
\nabla^{2} \vec{B}(r, z, \omega)+\frac{\omega^{2}}{c^{2}} \vec{B}(r, z, \omega)=-\mu_{0} B c \vec{\nabla} \times\left[\vec{z} \rho_{c}(r, z, \omega)\right], \\
\nabla^{2} \vec{E}(r, z, \omega)+\frac{\omega^{2}}{c^{2}} \vec{E}(r, z, \omega) \\
=\left(-i \mu_{0} \omega \beta c \hat{z}+\frac{1}{\epsilon_{0}} \vec{\nabla}\right) \rho_{c}(r, z, \omega)
\end{gathered}
$$

The Fourier time-transformed charge and current densities in Eqs. (6) and (7) are

$$
\begin{aligned}
\rho_{c}(r, z, \omega) & =\frac{\rho_{c}(r)}{\beta c} e^{i k_{z} z}, \\
j_{z}(r, z, \omega) & =\rho_{c}(r) e^{i k_{z} z},
\end{aligned}
$$

where $\omega=k_{z} v$ has been used and $k_{z}$ is the wave number in the direction of beam propagation.

As a consequence of the Fourier transformation, the fields will have the same $z$ dependence as the time-transformed sources $\rho_{c}(r, z, \omega)$ and $j_{z}(r, z, \omega)$ such that

$$
\begin{aligned}
& \vec{E}(r, z, \omega)=\vec{E}(r, \omega) e^{i k_{z} z}, \\
& \vec{B}(r, z, \omega)=\vec{B}(r, \omega) e^{i k_{z} z} .
\end{aligned}
$$

Adopting cylindrical coordinates the only nonvanishing excited field components by the beam in the cylindrical pipe due to the rotational symmetry are $E_{z}(r, \omega), E_{r}(r, \omega)$, and $B_{\theta}(r, \omega)$. 
For a uniformly charged thin disk of radius $a$ and charge $Q$, the density distribution in the transverse direction is

$$
\rho_{c}(r)=\frac{Q}{\pi a^{2}},
$$

where $a$ is the beam radius. Introducing $\gamma^{-2}=1-\beta^{2}$, the field components obey the following equations:

$$
\left[\frac{d^{2}}{d r^{2}}+\frac{1}{r} \frac{d}{d r}-\frac{k_{z}^{2}}{\gamma^{2}}\right]\left\{\begin{array}{l}
E_{z}(r, \omega) \\
E_{r}(r, \omega) \\
B_{\theta}(r, \omega)
\end{array}\right\}=\left\{\begin{array}{c}
i \frac{Q}{\pi a^{2}} \frac{k_{z}}{\epsilon_{0} \gamma^{2} \beta c} \\
0 \\
0
\end{array}\right\}
$$

The general solution for the $z$ component of the electric field is

$$
E_{z}(r, \omega)=\left\{\begin{array}{cc}
A_{1} I_{0}(\sigma r)+A_{2} K_{0}(\sigma r), & r>a \\
A_{3} I_{0}(\sigma r)-i \frac{Q}{\pi a^{2} \epsilon_{0} k_{z} \beta c}, & r \leqslant a
\end{array}\right.
$$

where $\sigma=k_{z} / \gamma=\omega / \beta c \gamma$, and $I_{0}$ and $K_{0}$ are modified Bessel functions of first and second kind, respectively, and $A_{1}, A_{2}$, and $A_{3}$ are constants to be determined by the boundary conditions.

To account for large but finite pipe wall conductivity, an impedance boundary condition will be used instead. The metallic surface exhibits a surface impedance $Z_{m}$ with equal resistive and inductive parts given by

$$
Z_{m}=\frac{1+i}{\sigma_{w} \delta_{s}}, \quad \delta_{s}=\sqrt{\frac{2}{\mu \omega \sigma_{w}}},
$$

where $\sigma_{w}$ is the wall conductivity and $\delta_{s}$ is the skin depth. Further, we assume that $\delta_{s}$ is very small compared with the wall thickness. At the surface, a surface current exists and the relation between this current and the electric field tangent to the surface is

$$
\vec{E}_{t}=Z_{m} \vec{j}_{s}=Z_{m} \hat{n} \times \vec{H}
$$

The field $\vec{E}_{t}$ in Eq. (15) is confined essentially to within the skin depth $\delta_{s}$ of the conducting medium forming the boundary surface. The numerical work required to find the propagation constants and the fields of the guide modes is a tedious job when the waveguide is bounded by a finite conducting medium. Fortunately, the perturbation that the finite conductivity of the bounding surface introduces into the problem under consideration is usually small. This allows application of a boundary-condition perturbation to obtain an estimate of the effect of the finiteness of the conductivity of the bounding surface. The corresponding impedance boundary condition at the bounding pipe wall is given in Eq. (15).

Upon using the continuity conditions of $E_{z}$ and $B_{\theta}$ at $r$ $=a$, as well as the boundary condition of a finite conductivity or finite surface current density at the pipe wall $r=b$, the excited fields become

$$
\begin{gathered}
\phi(r, z, \omega)=\frac{i Q e^{i k_{z} z}}{\pi a \epsilon_{0} \gamma k_{z} \beta c}\left\{\begin{array}{cc}
D_{1} I_{1}(\sigma a) I_{0}(\sigma r)-i I_{1}(\sigma a) K_{0}(\sigma r), & r>a \\
D_{1} I_{1}(\sigma a) I_{0}(\sigma r)+i\left[K_{1}(\sigma a) I_{0}(\sigma r)-\frac{1}{\sigma a}\right], & r \leqslant a
\end{array}\right. \\
E_{z}(r, z, \omega)=\frac{Q e^{i k_{z} z}}{\pi a \epsilon_{0} \gamma \beta c}\left\{\begin{array}{c}
D_{1} I_{1}(\sigma a) I_{0}(\sigma r)-i I_{1}(\sigma a) K_{0}(\sigma r), \quad r>a \\
D_{1} I_{1}(\sigma a) I_{0}(\sigma r)+i\left[K_{1}(\sigma a) I_{0}(\sigma r)-\frac{1}{\sigma a}\right], \quad r \leqslant a
\end{array}\right. \\
B_{\theta}(r, z, \omega)=-i \frac{Q e^{i k_{z} z}}{\pi a \epsilon_{0} c^{2}} \begin{cases}D_{1} I_{1}(\sigma a) I_{1}(\sigma r)+i I_{1}(\sigma a) K_{1}(\sigma r), & r>a \\
D_{1} I_{1}(\sigma a) I_{1}(\sigma r)+i K_{1}(\sigma a) I_{1}(\sigma r), & r \leqslant a\end{cases} \\
E_{r}(r, z, \omega)=-i \frac{Q e^{i k_{z} z}}{\pi a \epsilon_{0} \beta c} \begin{cases}D_{1} I_{1}(\sigma a) I_{1}(\sigma r)+i I_{1}(\sigma a) K_{1}(\sigma r), & r>a \\
D_{1} I_{1}(\sigma a) I_{1}(\sigma r)+i K_{1}(\sigma a) I_{1}(\sigma r), & r \leqslant a\end{cases}
\end{gathered}
$$

where $D_{1}$ is given by

$$
D_{1}=\frac{\left(\beta \gamma Z_{m} / c \mu_{0}\right) K_{1}(\sigma b)+i K_{0}(\sigma b)}{I_{0}(\sigma b)+i\left(\beta \gamma Z_{m} / c \mu_{0}\right) I_{1}(\sigma b)} .
$$

The fields for a pipe of infinite wall conductivity $\sigma_{w} \rightarrow \infty$, are obtained by substitution $Z_{m}=0$ in the above equations. For an ideal conductor with $Z_{m}=0$, we have $D_{1}$ $=i\left[K_{0}(\sigma b) / I_{0}(\sigma b)\right]$ and the electric-field component tangential to the pipe surface $E_{z}$ vanishes identically at $r=b$. 


\section{LONGITUDINAL IMPEDANCE FOR A WALL OF INFINITE CONDUCTIVITY}

Assuming a linear media such that $\epsilon$ and $\mu$ are independent of $E$ and $H$, then the Maxwell's equations in the presence of both an electric $\left(\vec{J}_{e}\right)$ and a fictitious magnetic $\left(\vec{J}_{m}\right)$ current density become [4]

$$
\begin{aligned}
\vec{\nabla} \times \vec{E}(\vec{r}, \omega) & =i \mu \omega \vec{H}(\vec{r}, \omega)-\vec{J}_{m}(\vec{r}, \omega), \\
\vec{\nabla} \times \vec{H}(\vec{r}, \omega) & =-i \omega \epsilon \vec{E}(\vec{r}, \omega)+\vec{J}_{e}(\vec{r}, \omega),
\end{aligned}
$$

where $\vec{J}_{e}$ and $\vec{J}_{m}$ are measured in $\mathrm{A} / \mathrm{m}^{2}$ and $\mathrm{V} / \mathrm{m}^{2}$, respectively. In calculating the coupling impedance for a beam pipe with a small hole in its perfectly conducting wall, we have both the electric and the magnetic wall boundary conditions. The electric wall boundary condition corresponds to $\vec{J}_{m}=0$ and a vanishing tangential electric field on the wall of the beam pipe $E_{z}(r=b)=0$. On the other hand, the tangential component of the magnetic field vanishes at the magnetic wall region where $\vec{J}_{e}=0$. Such a magnetic wall boundary does not really exist in practice, but may be approximated by a corrugated surface.

Multiplying Eq. (21) by $\vec{H}^{*}$ and the complex conjugate of Eq. (22) by $\vec{E}$, and then integrating them over the volume $V$ containing the sources yields

$$
\begin{aligned}
\int_{V} d^{3} x\left(\vec{E} \cdot \vec{J}_{e}^{*}+\vec{H}^{*} \cdot \vec{J}_{m}\right)= & i \omega \int_{V} d^{3} x\left(\mu|\vec{H}|^{2}-\epsilon^{*}|\vec{E}|^{2}\right) \\
& -\int_{S} d \vec{S} \cdot\left(\vec{E} \times \vec{H}^{*}\right)
\end{aligned}
$$

Since we have a smooth cylindrical beam pipe with no magnetic currents, the coupling impedance is defined in terms of the total work done by the fields as follows:

$$
\begin{aligned}
Z_{\|}(r, \omega) & =\frac{1}{Q^{2}} \int_{V_{\text {beam }}} d^{3} r^{\prime} \vec{E}\left(\vec{r}^{\prime}, \omega\right) \cdot \vec{J}_{e}^{*}\left(\vec{r}^{\prime}, \omega\right) \\
& =\frac{1}{Q^{2}} \int_{V_{\text {beam }}} d^{3} r^{\prime} E_{z}\left(r^{\prime}, z, \omega\right) j^{*}\left(r^{\prime}, z, \omega\right) .
\end{aligned}
$$

Using $J_{e}=j(r, z, \omega)=\left(Q / \pi a^{2}\right) e^{i k_{z} z}$ and $Z_{m}=0$, the impedance at any point $r \leqslant a$ from the beam axis for a pipe of infinite wall conductivity is

$$
\begin{aligned}
Z_{\|}(r, \omega)= & \frac{-i L}{\pi a^{2} \epsilon_{0} k_{z} \beta c}\left[\frac{r^{2}}{a^{2}}-\frac{2 r}{a} I_{1}(\sigma r)\left\{K_{1}(\sigma a)\right.\right. \\
& \left.\left.+\frac{K_{0}(\sigma b)}{I_{0}(\sigma b)} I_{1}(\sigma a)\right\}\right]
\end{aligned}
$$

where $L=2 \pi R$ is the ring circumference. Introducing the harmonic number $n=k_{z} R$ and $\chi_{0}=Z_{0} / 2 \beta \gamma^{2}$, Eq. (25) at $r$ $=a$ becomes

$$
Z_{\|}(r=a, \omega) \equiv Z_{\|}(\omega)=-i n \chi_{0} g_{\text {exact }}\left(a, b, k_{z}, \beta\right),
$$

$$
\begin{aligned}
g_{\text {exact }}\left(a, b, k_{z}, \beta\right)= & \frac{4 \gamma^{2}}{k_{z}^{2} a^{2}}\left[1-2 I_{1}(\sigma a)\left\{K_{1}(\sigma a)\right.\right. \\
& \left.\left.+\frac{K_{0}(\sigma b)}{I_{0}(\sigma b)} I_{1}(\sigma a)\right\}\right]
\end{aligned}
$$

where $Z_{0}=\sqrt{\mu_{0} / \epsilon_{0}}=377.0 \Omega$ is the vacuum impedance and $g\left(a, b, k_{z}, \beta\right)$ is the generalized geometry factor. The total longitudinal impedance for a pipe of an infinite wall conductivity and of a smooth surface is a negative imaginary constant.

We approximate the geometry factor in Eq. (27) by the following fitting formula:

$$
\begin{gathered}
Z_{\|}^{\text {approx }}(\omega)=-i n \chi_{0} g_{\text {approx }}\left(a, b, k_{z}, \beta\right), \\
g_{\text {approx }}\left(a, b, k_{z}, \beta\right)=\frac{g_{0}}{1+y^{2} n^{2} \alpha^{2}}, \\
g_{0}=x+2 \log \frac{b}{a}, \quad \alpha=\frac{a}{2 R} \sqrt{g_{0}} .
\end{gathered}
$$

Bisognano proposed a fitting formula for the expression of the ratio of the potential to the density, which is exactly our expression for the geometry factor of the longitudinal coupling impedance of a pipe of infinite wall conductivity in Eq. (27) [23] when we substitute in Bisognano's fitting formula $\gamma=1$. It is found that the factor

$$
\frac{1+2 \log (b / a)}{1+\left(k_{z}^{2} a^{2} / 4\right)[1+2 \log (b / a)]}
$$

in Bisognano's fitting formula is to be replaced by the factor $g_{\text {approx }}\left(a, b, k_{z}, \beta\right)$ in Eq. (29).

Transforming Bisognano's factor into the laboratory frame by the usually used replacement of the wave number $k_{z}$ by $k_{z} \gamma^{-1}$, will not fit the exact expression in Eq. (27) (see Fig. 5). Rather than calculating the impedance with the field on the axis, for which the parameter $1+2 \log (b / a)$ usually is used, we calculated the impedance by averaging over the transverse beam distribution and therefore, we find the best fit by introducing the two parameters $x(0 \leqslant x \leqslant 0.5)$ and $y$. The fitting parameter $y$ changes with changing the beam pipe geometry and is strongly dependent on the beam energy $(\beta)$.

\section{TOTAL IMPEDANCE FOR FINITE WALL CONDUCTIVITY}

Upon substituting the electric field $E_{z}(r, z, \omega)$ from Eq. (17) into Eq. (24), the total longitudinal coupling impedance at any point $r \leqslant a$ of a pipe of finite wall conductivity is

$$
\begin{aligned}
Z_{\|}(r, \omega)= & \frac{2 L r}{\pi a^{3} \epsilon_{0} k_{z} \beta c}\left[D_{1} I_{1}(\sigma a) I_{1}(\sigma r)\right. \\
& \left.+i\left(K_{1}(\sigma a) I_{1}(\sigma r)-\frac{r}{2 a}\right)\right] .
\end{aligned}
$$

At the beam surface $r=a$, the total impedance $Z_{\|}(r=a, \omega)$ $\equiv Z_{\|}(\omega)$ in Eq. (31) reduces into 


$$
\begin{aligned}
Z_{\|}(\omega)= & -i \frac{L}{\pi a^{2} \epsilon_{0} k_{z} \beta c} \\
& \times\left\{1-2 I_{1}(\sigma a)\left[K_{1}(\sigma a)-i D_{1} I_{1}(\sigma a)\right]\right\} \\
= & -i \frac{L}{\pi a^{2} \epsilon_{0} k_{z} \beta c}\left(1-2 I_{1}(\sigma a)\right. \\
& \left.\times\left[K_{1}(\sigma a) \frac{K_{0}(\sigma b)}{I_{0}(\sigma b)} I_{1}(\sigma a)\right]\right) \\
& +\frac{2 L I_{1}^{2}(\sigma a)}{\pi a^{2} \epsilon_{0} k_{z} \beta c}\left(D_{1}-i \frac{K_{0}(\sigma b)}{I_{0}(\sigma b)}\right) \\
= & z_{\|}^{\mathrm{spch}}(\omega)+Z_{\|}^{\mathrm{rw}}(\omega) .
\end{aligned}
$$

The first term on the right-hand side of Eq. (32) is the spacecharge impedance for a perfectly conducting pipe wall, whereas the second term accounts for the resistive wall impedance when the pipe wall has a large but finite surface impedance. For a perfectly conducting wall with $\sigma_{w} \rightarrow \infty$ or $Z_{m} \rightarrow 0$, we have $D_{1}=i\left[K_{0}(\sigma b) / I_{0}(\sigma b)\right]$, and the second term on the right-hand side of Eq. (32) vanishes, and the total impedance reduces into Eq. (25).

Upon substitution for $Z_{m}$ from Eq. (14) and using $n$ $=k_{z} R$, the resistive wall impedance $Z_{\|}^{\mathrm{rw}}(\omega)$ becomes

$$
\begin{gathered}
Z_{\|}^{\mathrm{rw}}(\omega)=R_{\|}^{\mathrm{rw}}(\omega)+i \chi_{\|}^{\mathrm{rw}}(\omega), \\
R_{\|}^{\mathrm{rw}}(\omega)=R_{0} \frac{4(\sigma a)^{-2} I_{1}^{2}(\sigma a)}{\left[I_{0}^{2}(\sigma b)-2 \alpha I_{0}(\sigma b) I_{1}(\sigma b)+2 \alpha^{2} I_{1}^{2}(\sigma b)\right]},
\end{gathered}
$$

$\chi_{\|}^{\mathrm{rw}}(\omega)$

$$
=\chi_{0} \frac{4(\sigma a)^{-2} I_{1}^{2}(\sigma a)\left[I_{0}(\sigma b)-2 \alpha I_{1}(\sigma b)\right]}{I_{0}(\sigma b)\left[I_{0}^{2}(\sigma b)-2 \alpha I_{0}(\sigma b) I_{1}(\sigma b)+2 \alpha^{2} I_{1}^{2}(\sigma b)\right]},
$$

$$
R_{0}=\chi_{0}=\frac{n Z_{0} \beta \delta_{s}^{*}}{2 \sqrt{n} b}
$$

where $R_{0}$ and $\chi_{0}$ are the components of the resistive wall impedance in the long-wavelength limit, and $\delta_{s}^{*}$ $=\sqrt{2 / \mu_{0} \omega_{0} \sigma_{w}}$ is the skin depth at the revolution frequency $\omega_{0}=\beta c / R$, and $\alpha=\beta \gamma / \mu_{0} \sigma_{w} \delta_{s} c=\left(\beta^{2} \gamma / 2\right) k_{z} \delta_{s}$.

For very small $\alpha$ values corresponding to the condition $k_{z} \delta_{s} \ll 2 / \beta^{2} \gamma$, which mostly satisfied, the resistive wall impedance becomes

$$
\begin{aligned}
Z_{\|}^{\mathrm{rw}}(\omega) & \approx(1+i) \frac{n Z_{0} \beta \delta_{s}^{*}}{2 \sqrt{n} b} \frac{4 I_{1}^{2}(\sigma a)}{\sigma^{2} a^{2} I_{0}^{2}(\sigma b)} \\
& \equiv(1+i) \frac{n Z_{0} \beta \delta_{s}^{*}}{2 \sqrt{n} b} g^{\mathrm{rw}}
\end{aligned}
$$

where $g^{\mathrm{rw}}$ is an effective resistive wall geometry factor. In the relativistic limit $\sigma_{w} \rightarrow 0$ we have $I_{1}(\sigma a)=0.5 \sigma a$ and therefore Eq. (37) reduces into the well-known expression for the resistive wall impedance of ultrarelativistic particle beams $[7,14]$.

We now introduce another approach for calculating the resistive wall impedance using the flux of the Poynting vector at the pipe wall. The axial component of the electric field at the pipe wall gives rise to a Poynting vector component directed into the pipe wall. This accounts for a power loss in the pipe wall, which we express in terms of the coupling impedance defined as follows:

$$
\begin{aligned}
Z_{\|}^{\mathrm{rw}}(\omega) & =2 \pi b L \frac{S(r=b, \omega)}{Q^{2}} \\
& =\frac{2 \pi b L}{Q^{2}}\left[E_{z}(r=b, \omega) H_{\theta}^{*}(r=b, \omega)\right] .
\end{aligned}
$$

Upon using Eqs. (17) and (18) we find the following expressions for the flux of the Poynting vector at the pipe wall ( $r$ $=b$ ) and for the corresponding resistive wall impedance:

$$
\begin{aligned}
S(r=b, \omega)= & \frac{Q^{2}}{\pi^{2} b^{2} \sigma_{w} \delta_{s} \sigma^{2} a^{2}} \\
& \times \frac{I_{1}^{2}(\sigma a)(1+i)}{I_{0}^{2}(\sigma b)-2 \alpha I_{1}(\sigma b) I_{0}(\sigma b)+2 \alpha^{2} I_{1}^{2}(\sigma b)}, \\
Z_{\|}^{\mathrm{rw}}(\omega)= & (1+i) \frac{R}{b \sigma_{w} \delta_{s}} \\
& \times \frac{4 I_{1}^{2}(\sigma a) / \sigma^{2} a^{2}}{I_{0}^{2}(\sigma b)-2 \alpha I_{1}(\sigma b) I_{0}(\sigma b)+2 \alpha^{2} I_{1}^{2}(\sigma b)},
\end{aligned}
$$

where $R$ is the ring radius. Using $Z_{0}=\mu_{0} c$ and $n=k_{z} R$ the resistive wall impedance in Eq. (40) becomes

$$
\begin{aligned}
Z_{\|}^{\mathrm{rw}}(\omega)= & \frac{n Z_{0} \beta \delta_{s}(1+i)}{2 b} \\
& \times \frac{4\left[I_{1}^{2}(\sigma a) / \sigma^{2} a^{2}\right]}{I_{0}^{2}(\sigma b)-2 \alpha I_{1}(\sigma b) I_{0}(\sigma b)+2 \alpha^{2} I_{1}^{2}(\sigma b)} .
\end{aligned}
$$

Introducing $\delta_{s}^{*}=\sqrt{2 / \mu_{0} \omega_{0} \sigma_{w}}$ as the skin depth at the revolution frequency and using $\alpha \ll 1$, Eq. (41) becomes

$$
Z_{\|}^{\mathrm{rw}}(\omega) \approx(1+i) \frac{n Z_{0} \beta \delta_{s}^{*}}{2 \sqrt{n} b} \frac{4 I_{1}^{2}(\sigma a)}{\sigma^{2} a^{2} I_{0}^{2}(\sigma b)} .
$$

We see that the real and imaginary parts of the resistive wall impedance in Eq. (40) are equal. Contrary to the Poynting vector approach, the components of the resistive wall impedance are generally not equal [see Eqs. (34) and (35)] according to the general approach based on the impedance definition in Eq. (24). For small $\alpha$ values such that $k_{z} \delta_{s} \ll 2 / \beta^{2} \gamma$ they do become approximately equal. 


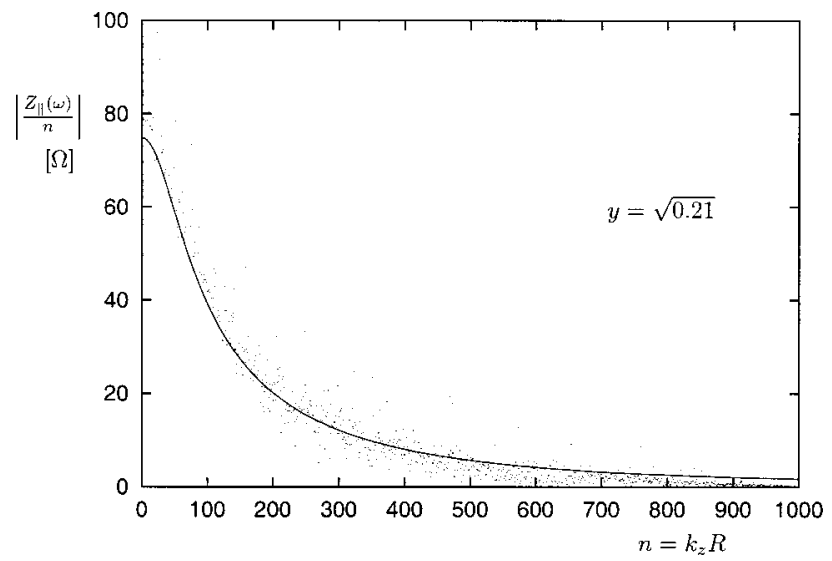

FIG. 1. Space-charge impedance seen by the beam for $a$ $=0.2 b, \sigma_{w}=\infty, L=20 \mathrm{~m}, b=0.314 \mathrm{~m}$, and $\beta=0.9481$. The dots come from the numerical simulation with PATRIC, the solid line comes from Eq. (28).

The power flow across the pipe surface is the sum of the conduction loss in the wall $\left(P_{L}\right)$ and the difference between the power stored in the magnetic and electric fields $\left[2 i \omega\left(W_{m}-W_{e}\right)\right][2]$,

$$
P=2 i \omega\left(W_{m}-W_{e}\right)+P_{L}
$$

For a perfectly conducting wall the power flow across the pipe surface vanishes, since $P_{L}=0$ and the average electric and magnetic energies associated with a given mode are equal, $W_{m}=W_{e}$. The physical reason for the appearance of the second term in $\chi^{\mathrm{rw}}(\omega)$ in Eq. (35) is due to the fact that the resistive wall impedance in Eq. (33) was evaluated by integrating over the transverse beam distribution, and therefore it accounts for the difference between the power stored in the magnetic and electric fields, which is not the case when evaluating the resistive wall impedance using the value of the pointing vector at the wall of the pipe. For $k_{z} \delta_{s}$ $\ll 2 / \beta^{2} \gamma$, the stored power in the magnetic and electric fields are nearly equal and therefore, the resistive wall impedance can be approximated by the expression in Eq. (41).

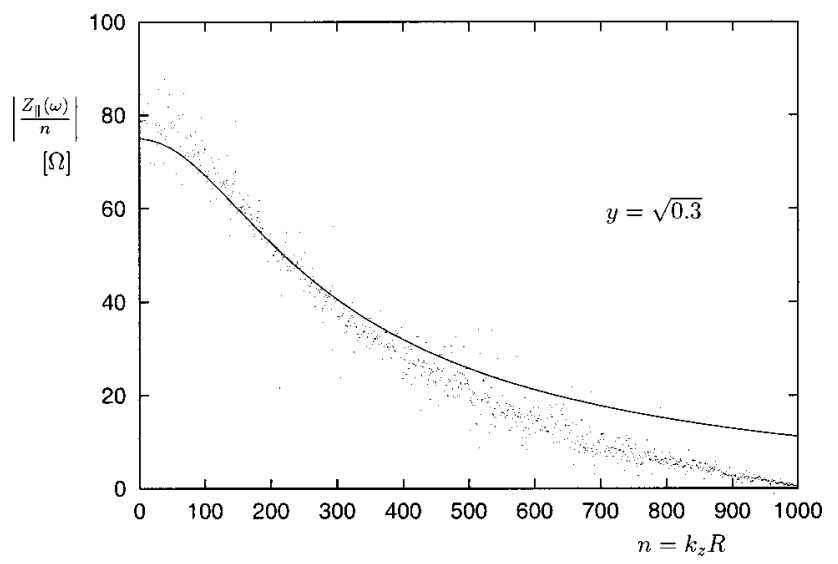

FIG. 2. Space-charge impedance seen by the beam for $a$ $=0.2 b, \sigma_{w}=\infty, L=20 \mathrm{~m}, b=0.1 \mathrm{~m}$, and $\beta=0.9481$. The dots come from the numerical simulation with PATRIC, the solid line comes from Eq. (28).

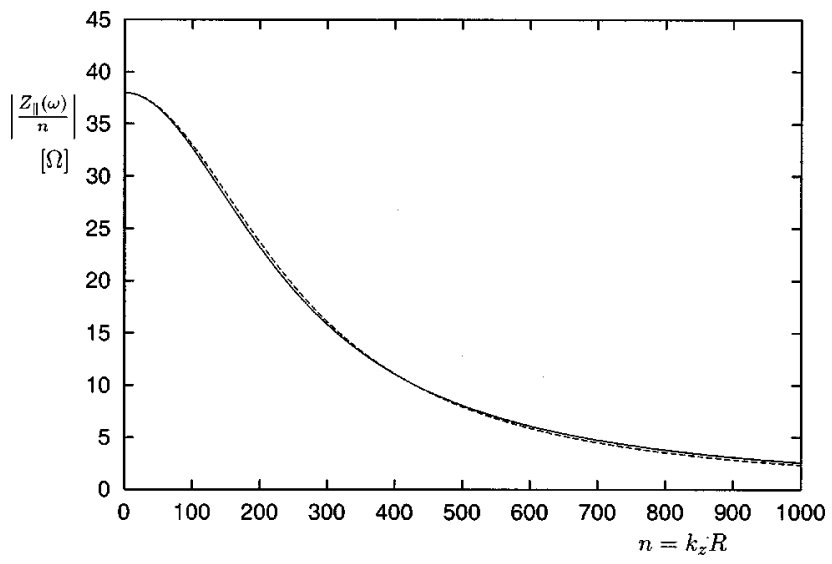

FIG. 3. Exact (solid line) and approximate (dotted line) coupling impedance according to Eqs. (26) and (28) for $a=0.5 b, \sigma_{w}=\infty$, $L=20 \mathrm{~m}, b=0.1 \mathrm{~m}$, and $\beta=0.948$. Fitting parameter $y=\sqrt{0.13}$.

\section{NUMERICAL ANALYSIS}

We have evaluated the space-charge impedance seen by the beam using the PATRIC code, and we have compared it with the fitting formula given in Eq. (28). In the simulations, we have let a bunch containing $10^{13}$ protons evolve over about $10 \mu$ s with no voltage applied. The only force on the beam particles comes from the space charge, and it is evaluated at each time step in the beam rest frame by solving the Poission equation on a grid of $2048 \times 32$ cells ( 2048 longitudinal cells and 32 radial cells, since we assume a cylindrical symmetry for our system beam perfectly conducting pipe). To transform the result of our computation into the lab frame, we have to scale down the electric field of a factor $\gamma^{2}$ and stretch its harmonic content of a factor $\gamma$. The bunch has been modeled as an ensemble of $10^{6}$ macroparticles. The impedance has been calculated in the following way: we have first taken the ratio between the Fourier transform of the self-induced field and the beam current spectrum at different subsequent instants spaced by a constant number of time steps (one time step is $\Delta t=100 \mathrm{~ns}$ ), and then we have averaged the result over all of them in order to reduce the fluctuations due to statistical noise. As expected, the resulting

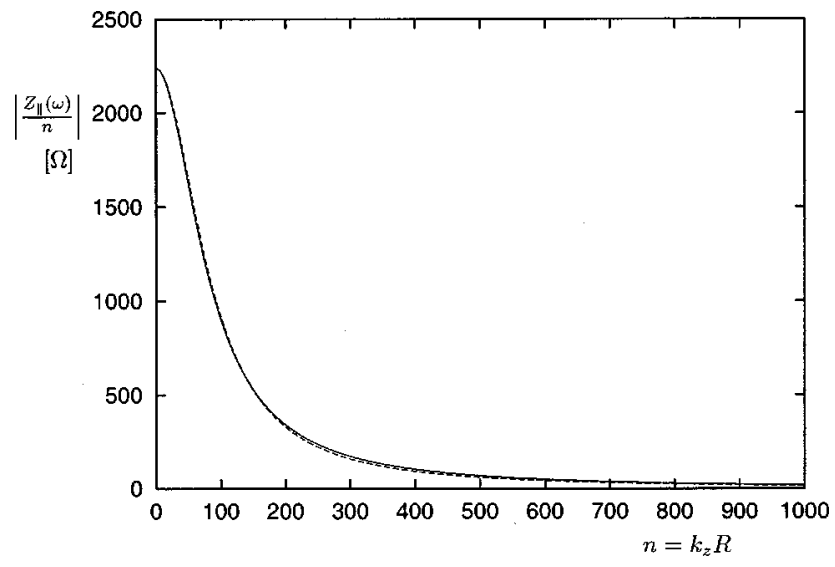

FIG. 4. Exact (solid line) and approximate (dotted line) coupling impedance according to Eqs. (26) and (28) for $a=0.5 b, \sigma_{w}=\infty$, $L=20 \mathrm{~m}, b=0.1 \mathrm{~m}$, and $\beta=0.155$. Fitting parameter $y=\sqrt{0.25}$. 


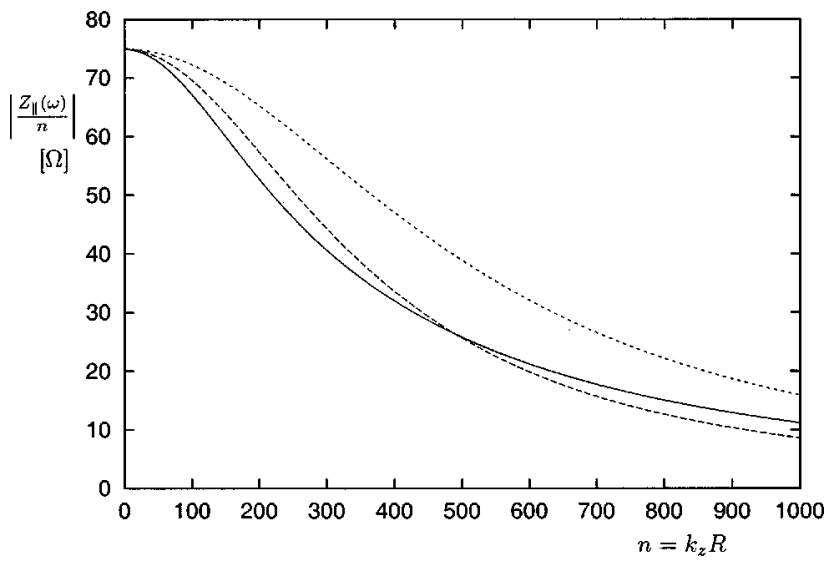

FIG. 5. Exact (solid line) and approximate (long dashed line) coupling impedance according to Eqs. (26) and (28) for $a=0.2 b$, $\sigma_{w}=\infty, L=20 \mathrm{~m}, b=0.1 \mathrm{~m}$, and $\beta=0.948$. The upper shortdashed line gives the coupling impedance according to the modified Bisognano formula in Eq. (44). Fitting parameter $y=\sqrt{0.21}$.

impedance has a dominant imaginary component, whereas its real part is pure fluctuation around the zero level.

In Figs. 1 and 2, we have plotted the reactance over the harmonic number as a function of the harmonic number, as is customary when referring to beam stability. The energy of the protons is $2 \mathrm{GeV}$, and the bunch is 10 -m long over a circumference length of $2 \pi R=20 \mathrm{~m}$. Beam radius and pipe radius have been chosen to be $a=0.2 b \mathrm{~m}$ and $b=0.314 \mathrm{~m}$, respectively, for the simulation that relates to Fig. 1, and $a$ $=0.2 b \mathrm{~m}$ and $b=0.1 \mathrm{~m}$, respectively, for Fig. 2. In this way, the ratio $b / a=5$ stays constant, which means that there is no difference in the macroscopic structure of the self-induced electric field between these two cases, but the spectral contents are obviously different. Taking a look at Figs. 1 and 2, we immediately see that the agreement between the modified fitting formula in Eq. (28) for the space-charge impedance and the numerically evaluated one is excellent.

In Figs. 3-5 we plotted the impedance per harmonic number $Z_{\|}(\omega) / n$ according to the exact and approximate expressions in Eqs. (26) and (28) as functions of the harmonic number $n=k_{z} R$ for various beam pipe parameters. We see from these figures an excellent agreement between the exact and the fitting formulas.

A fitting formula for the ratio of the Fourier-transformed potential and charge density was proposed by Bisognano in investigating solitary waves in nonrelativistic particle beams [23]. This ratio is exactly the expression for the geometry factor in Eq. (27) for the longitudinal coupling impedance of a pipe of infinite wall conductivity. The factor

$$
\frac{1+2 \log (b / a)}{1+\left(k_{z}^{2} a^{2} / 4\right)[1+2 \log (b / a)]}
$$

in Bisognano's fitting formula was used by Rumolo et al. to fit the space-charge impedance for arbitrary wavelengths [22]. It is found that the proposed fitting factor is replaced by the factor given in Eq. (29). Rather than using the field on the axis, Eq. (29) gives the $g$ factor when calculating the impedance by averaging over the transverse beam distribu-

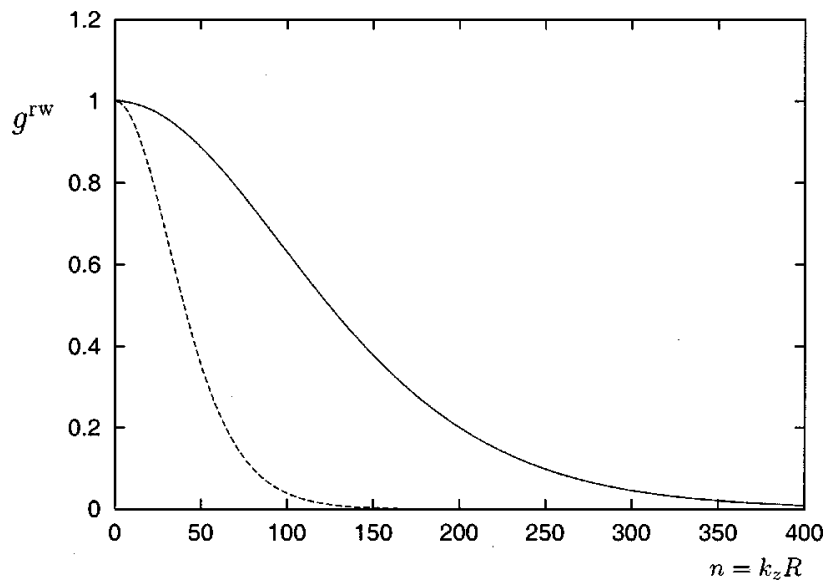

FIG. 6. Resistive wall geometry factor according to Eq. (37) for $\beta=0.948$ (solid line) and $\beta=0.155$ (dashed line) for $a=0.2 b, \sigma_{w}$ $=10^{6}(\Omega \mathrm{m})^{-1}, L=20 \mathrm{~m}$, and $b=0.1 \mathrm{~m}$.

tion. The fitting parameter $x$ is found to have a fixed value $(x=0.5)$ for all radii $a$ and $b$ and for any value of $\beta$, as expected $[10,13]$, whereas the parameter $y$ changes with changing $a$ and $b$ or $\beta$.

Accounting for the averaging over the transverse beam distribution and transforming into the laboratory frame suggests the use of the following modified Bisognano formula in the lab frame:

$$
g=\frac{g_{0}}{1+n^{2} / \gamma^{2} a^{2} / 4 R^{2}}, \quad g_{0}=0.5+2 \log \frac{b}{a} .
$$

In Fig. 5 we have plotted the exact and approximate coupling impedance according to Eqs. (26) (solid line) and (28) (long dashed line) for $a=0.2 b, \sigma_{w}=\infty, L=20 \mathrm{~m}, b=0.1 \mathrm{~m}$, and $\beta=0.948$. The upper short-dashed line gives the coupling impedance according to the modified Bisognano formula in Eq. (44). From Fig. 5, we immediately see the discrepancy between the result of our computation and the Lorentzian profile predicted by Bisognano.

The resistive wall impedance also has been investigated using the volume integral of the energy density over the transverse beam distribution and the flux of the Poynting vector at the pipe wall. In Figs. 6 and 7 we plotted the resistive wall geometry factor and resistive wall impedance per harmonic number according to Eq. (37) for $\beta=0.948$ (solid line) and $\beta=0.155$ (dashed line), respectively. We use the parameters $a=0.2 b, \quad \sigma_{w}=10^{6}(\Omega \mathrm{m})^{-1}, L=20 \mathrm{~m}$, and $b$ $=0.1 \mathrm{~m}$. We see that $Z^{r w}(\omega) / n$ is very small compared with the space-charge impedance, and it is larger for relativistic particle beams.

\section{CONCLUSIONS}

In this paper, the longitudinal space-charge and resistive wall impedances have been investigated in a smooth cylindrical beam pipe. In Sec. II, the derivation of the excited electromagnetic fields in a beam pipe of both infinite and finite wall conductivities has been presented. In Sec. III, the space-charge impedance for a pipe of infinite wall conductivity has been calculated, and a fitting formula with some 


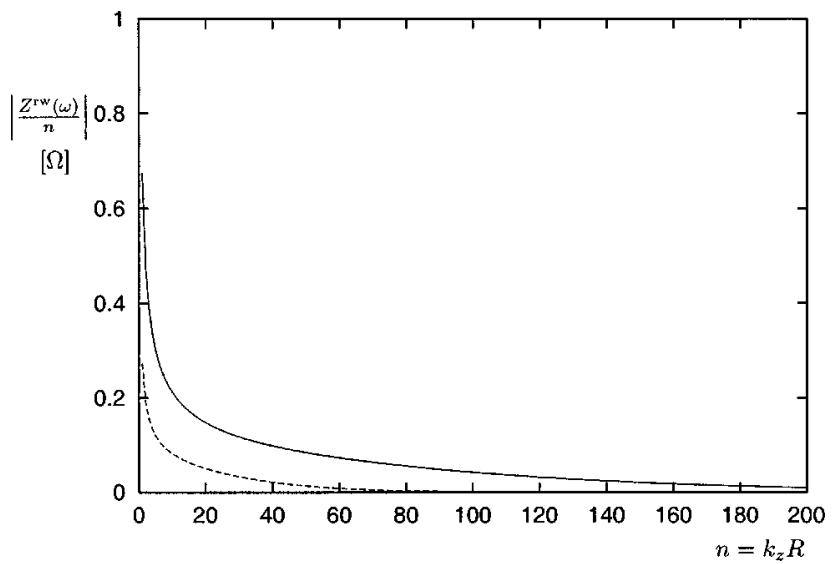

FIG. 7. Resistive wall impedance per harmonic number according to Eq. (37) for $\beta=0.948$ (solid line) and $\beta=0.155$ (dashed line) for $a=0.2 b, \sigma_{w}=10^{6}(\Omega \mathrm{m})^{-1}, L=20 \mathrm{~m}$ and $b=0.1 \mathrm{~m}$.

values for the fitting parameters of the corresponding generalized geometry factor have been given. For a pipe of finite wall surface impedance, the space-charge and resistive wall impedances have been investigated in Sec. IV, where we also presented a second approach for recalculating the resistive wall impedance using the flux of the Poynting vector at the wall of the beam pipe.

At any point $r \leqslant a$ from the beam axis, a general expression for the total coupling impedance has been obtained [see Eq. (31)]. At the beam surface $r=a$ and for a pipe of infinite wall conductivity $\left(Z_{m}=0\right)$, the expression for the total impedance in Eq. (31) reduces into the expression that was derived by Zotter and Kheifets in studying the impedance of uniform beams in concentric cylindrical wall chambers, when a single cylindrical chamber is considered [7].

A fitting formula for the space-charge impedance at the beam surface $(r=a)$ and for a pipe of infinite wall conductivity, which is valid for arbitrary wavelengths, has been obtained by averaging over the transverse beam distribution [Eqs. (28) and (29)], rather than using the field on the axis. Accounting for the averaging over the transverse beam distribution and transforming into the laboratory frame suggests the use of Eq. (44) as a modified Bisognano formula in the lab frame. Figure 5 shows the discrepancy between the result of computing the space-charge impedance using Eq. (28) and the Lorentzian profile predicted by Bisognano according to the modified formula in Eq. (44) [22,23].

The resistive wall impedance has been investigated using the volume integral of the energy density over the transverse beam distribution. An expression for the resistive wall impedance, which accounts for beams of finite sizes and for arbitrary velocity $\beta$, is given in Eq. (33). In the extreme relativistic limit $\beta \rightarrow 1$, Eq. (33) reduces into the well-known expression for the resistive wall impedance, which has equal real and imaginary parts and is independent of the beam size $a$. For $k_{z} \delta_{s} \ll 2 / \beta^{2} \gamma$, the resistive wall impedance in Eq. (33) can be approximated by the expression in Eq. (37). In this case, the expressions for resistive wall impedance in Eqs. (33) and (41) become approximately equal.
[1] A. A. Oliner, IEEE Trans. Microwave Theory Tech. 32, 1022 (1984).

[2] R. E. Collin, Field Theory of Guided Waves (McGraw-Hill, New York, 1960).

[3] R. E. Collin, Foundations of Microwave Engineering (McGraw-Hill, New York, 1966).

[4] David M. Pozar, Microwave Engineering (Addison-Wesley, Reading, MA, 1990).

[5] V. G. Vaccaro, CERN ISR-RF, 65-35 (1966).

[6] V. K. Neil and A. M. Sessler, Rev. Sci. Instrum. 36, 429 (1965).

[7] B. W. Zotter and S. A. Kheifets, Impedances and Wakes in High-Energy Particle Accelerators (World Scientific, Singapore, 1998), Chap. 6.

[8] B. Zotter, Part. Accel. 1, 311 (1972).

[9] S. S. Kurennoy, Part. Nuclei 24, 380 (1993).

[10] S. S. Kurennoy, in Workshop on Instabilities of High Intensity Hadron Beams in Rings, edited by T. Roser and S. Y. Zhang, AIP Conf. Proc. No. 496 (AIP, New York, 1999), p. 361.

[11] R. L. Gluckstern, J. van Zeijts, and B. Zotter, Phys. Rev. E 47, 656 (1993).

[12] R. L. Gluckstern and A. V. Fedotov, Workshop on Instabilities of High Intensity Hadron Beams in Rings, edited by T. Roser and S. Y. Zhang, AIP Conf. Proc. No. 496 (AIP, New York, 1999), p. 77.
[13] J. G. Wang, Workshop on Instabilities of High Intensity Hadron Beams in Rings, edited by T. Roser and S. Y. Zhang, AIP Conf. Proc. No. 496 (AIP, New York, 1999), p. 277.

[14] Jean-Louis Laclare, Cern Accelerator School (CAS), Vol. II, edited by P. Bryant and S. Turner, CERN 85-19 (1985), pp. 377-414.

[15] M. Reiser, Theory and Design of Charged Particle Beams (Wiley, New York, 1994).

[16] A. W. Chao, Physics of Collective Beam Instabilities in High Energy Accelerators (Wiley, New York, 1993).

[17] J. G. Wang, H. Suk, D. X. Wang, and M. Reiser, Phys. Rev. Lett. 72, 2029 (1994).

[18] A. G. Ruggiero and V. C. Vaccaro, CERN-Report No. ISRTH/68-33 (1968).

[19] I. Hofmann, G. Miano, and G. Rumolo, Proceedings of the European Particle Accelerator Conference, Stockholm, 1998, p. 2002.

[20] I. Hofmann, Laser Part. Beams 3, 1 (1985).

[21] I. Hofmann, Phys. Rev. E 57, 4713 (1998).

[22] G. Rumolo, G. Miano, C. Serpico, and I. Hofmann, Phys. Plasmas 6, 4349 (1999).

[23] Joseph J. Bisognano, Fifth European Particle Accelerator Conference (EPAC96), edited by S. Myers, A. Pacheco, R. Pascual, Ch. Petit-Jean-Genaz, and J. Poole (Institute of Physics, Bristol, 1996), Vol. 1, p. 328. 